\title{
Sexual Behavior Induction of c-Fos in the Nucleus Accumbens and Amphetamine-Stimulated Locomotor Activity Are Sensitized by Previous Sexual Experience in Female Syrian Hamsters
}

\author{
Katherine C. Bradley ${ }^{1}$ and Robert L. Meisel ${ }^{2}$ \\ ${ }^{1}$ Graduate Neuroscience Program, and '2Department of Psychological Sciences, Purdue University, West Lafayette, \\ Indiana 47907-1364
}

Dopamine transmission in the nucleus accumbens can be activated by drugs, stress, or motivated behaviors, and repeated exposure to these stimuli can sensitize this dopamine response. The objectives of this study were to determine whether female sexual behavior activates nucleus accumbens neurons and whether past sexual experience cross-sensitizes neuronal responses in the nucleus accumbens to amphetamine. Using immunocytochemical labeling, c-Fos expression in different subregions (shell vs core at the rostral, middle, and caudal levels) of the nucleus accumbens was examined in female hamsters that had varying amounts of sexual experience. Female hamsters, given either 6 weeks of sexual experience or remaining sexually naive, were tested for sexual behavior by exposure to adult male hamsters. Previous sexual experience increased c-Fos labeling in the rostral and caudal levels but not in the middle levels of the nucleus accumbens. Testing for sexual behavior increased labeling in the core, but not the shell, of the nucleus accumbens. To validate that female sexual behavior can sensitize neurons in the mesolimbic dopamine pathway, the locomotor responses of sexually experienced and sexually naive females to an amphetamine injection were then compared. Amphetamine increased general locomotor activity in all females. However, sexually experienced animals responded sooner to amphetamine than did sexually naive animals. These data indicate that female sexual behavior can activate neurons in the nucleus accumbens and that sexual experience can cross-sensitize neuronal responses to amphetamine. In addition, these results provide additional evidence for functional differences between the shell and core of the nucleus accumbens and across its anteroposterior axis.

Key words: female sexual behavior; nucleus accumbens; shell; core; c-Fos; sensitization; cross-sensitization; amphetamine
Dopamine neurons originating in the midbrain ventral tegmental area and projecting to various forebrain nuclei, including the nucleus accumbens, are part of the mesolimbic dopamine system. It has been suggested that this dopamine system is important for the regulation of appetitive behaviors (Mitchell and Gratton, 1994; Salamone, 1994, 1996; Ikemoto and Panksepp, 1999), as well as self-administration of drugs of abuse (Pierre and Vezina, 1998; Koob, 1999; Lorrain et al., 1999; McKinzie et al., 1999; Peoples et al., 1999; Bradberry et al., 2000). Systemic administration of a variety of drugs of abuse (e.g., cocaine, amphetamine, and heroin) activates dopamine pathways (Pontieri et al., 1995; Nisell et al., 1997; Pierce and Kalivas, 1997a; Tanda et al., 1997; Tanda and Di Chiara, 1998; Barrot et al., 1999; Cadoni and Di Chiara, 1999), and repeated exposure to these pharmacological agents can sensitize these dopamine-responsive neurons (Robinson et al., 1988; Kalivas et al., 1992; Kalivas and Duffy, 1993; Pierce and Kalivas, 1995; Kuczenski et al., 1997; Nisell et al., 1997; Birrell and Balfour, 1998; Heidbreder and Feldon, 1998; Cadoni and Di Chiara, 1999; Cadoni et al., 2000). Research has provided evidence that the nucleus accumbens also responds to certain properties associated with mating. Extracellular dopa-

\footnotetext{
Received June 8, 2000; revised Dec. 12, 2000; accepted Dec. 20, 2000.

This research was supported by National Science Foundation Grant IBN-9723876. We thank Melissa Zila, Shannon McCanna, Marchelle Baker, Michael Huntington, and Deborah Shelley for their expert assistance with the behavioral testing and c-Fos processing.

Correspondence should be addressed to Dr. Robert L. Meisel, Department of Psychological Sciences, Purdue University, West Lafayette, IN 47907-1364. E-mail: meisel@psych.purdue.edu.

Copyright (C) 2001 Society for Neuroscience $\quad 0270-6474 / 01 / 212123-08 \$ 15.00 / 0$
}

mine levels in the nucleus accumbens increase during sexual interactions in female rats (Mermelstein and Becker, 1995; Pfaus et al., 1995) and hamsters (Meisel et al., 1993; Kohlert et al., 1997; Kohlert and Meisel, 1999). Similar to repeated drug administration, multiple sexual behavior tests also augment the increases in nucleus accumbens dopamine levels, suggesting that sexual experience can sensitize neurons in the dopamine pathway (Kohlert and Meisel, 1999).

The nucleus accumbens is composed of many anatomically distinct subregions, the most familiar of which are the shell and the core. Anatomical connections of the shell and core diverge, suggesting that these two subregions regulate different functions (Crawley et al., 1985a,b; Heimer et al., 1991; Zahm and Brog, 1992; Brog et al., 1993; Kalivas and Duffy, 1995; MaldonadoIrizarry et al., 1995; Pierce and Kalivas, 1995; Pontieri et al., 1995; Broening et al., 1997; Heimer et al., 1997; Kelley et al., 1997; Stratford and Kelley, 1997; Heidbreder and Feldon, 1998; Lanca et al., 1998; Bassareo and Di Chiara, 1999; Di Chiara et al., 1999b; Groenewegen et al., 1999; Kelley, 1999; McKinzie et al., 1999; Zahm, 1999; Brown and Molliver, 2000). Because the nucleus accumbens is a heterogeneous nucleus, it is not clear whether responses to female sexual behavior are localized to specific subregions of the nucleus accumbens or spread throughout the entire nucleus. Techniques previously used to answer this question (e.g., microdialysis) are not spatially sensitive enough to explore the functional heterogeneity of the accumbens. In contrast, immunocytochemical processing for the c-Fos protein provides a method for examining discrete cellular activation among subregions of the nucleus accumbens. Thus, the first purpose of 
this experiment was to determine whether cellular activation after female sexual behavior is localized to specific subregions of the nucleus accumbens.

An interesting property of these dopamine pathways is crosssensitization. In other words, the dopamine neurons previously sensitized to one drug will exhibit sensitized responses to another drug given for the first time (Cunningham and Kelley, 1992; Pierce and Kalivas, 1997a; Birrell and Balfour, 1998; Taylor and Horger, 1999). In addition to cross-sensitization among drugs, several studies have reported cross-sensitization between repeated exposures to pharmacological agents and natural motivated behaviors (Mitchell and Stewart, 1990a,b; Tidey and Miczek, 1997; Fiorino and Phillips, 1999). Therefore, we examined whether sexually experienced and sexually naive animals would respond differently to a novel stimulus known to activate dopamine pathways (i.e., cross-sensitization), such as amphetamine. If female sexual behavior sensitizes dopamine pathways, then sexually experienced females should display an augmented behavioral response to a single injection of amphetamine.

\section{MATERIALS AND METHODS}

\section{General methods}

Animals. Male and female Syrian hamsters were delivered from Charles River Laboratories (Kingston, NY) at $\sim 60 \mathrm{~d}$ of age. The females were housed individually, and male stimulus animals were housed in groups of three or four in plastic cages $(50.8 \times 40.6 \times 20.3 \mathrm{~cm})$. The animal colony room was maintained at a constant temperature $\left(22^{\circ} \mathrm{C}\right)$ with the lights off between 1:30 and 11:30 P.M. (14/10 hr light/dark cycle). Food and water were available ad libitum.

The procedures used in this experiment are in accordance with National Institutes of Health Guidelines for the Care and Use of Laboratory Animals and have been approved by the Purdue Animal Care and Use Committee.

Sexual experience. Approximately 1 week after the females arrived at the laboratory, they were bilaterally ovariectomized under sodium pentobarbital (Nembutal) anesthesia (8.5 mg per 100 gm body weight, i.p.). After ovariectomy, the females were initially divided into two groups. One group of females received 6 weeks of sexual experience with a stimulus male; the second group remained sexually naive. All of the females were hormonally primed once a week for the 6 week period. At both 48 and $24 \mathrm{hr}$ before the sexual experience, the females were injected subcutaneously with $10 \mu \mathrm{g}$ of estradiol benzoate in $0.1 \mathrm{ml}$ of cottonseed oil. On the day of the experience test, the females received $500 \mu \mathrm{g}$ of progesterone in $0.1 \mathrm{ml}$ of cottonseed oil (subcutaneous injection). Females not receiving sexual experience were injected with the hormone regimen and remained in their home cages in the colony room. At 4-5 hr after the administration of progesterone, an adult male hamster that had received sexual experience through use in other sexual behavior studies was placed in the home cage of the experimental female. The order of the cages containing the males was rotated each week to minimize the likelihood that an individual male and female were paired more than once during the 6 weeks of sexual experience.

Immunocytochemistry. Female hamsters, killed with an overdose of sodium pentobarbital, were intracardially perfused with a $25 \mathrm{~mm}$ PBS solution, $\mathrm{pH} 7.5$, for $2 \mathrm{~min}$ (flow rate, $25 \mathrm{ml} / \mathrm{min}$ ), followed by $4 \%$ paraformaldehyde in PBS for 20 min. Brains were post-fixed for $2 \mathrm{hr}$ in the paraformaldehyde and stored in $10 \%$ sucrose PBS overnight at $4^{\circ} \mathrm{C}$.

Serial coronal $40 \mu \mathrm{m}$ frozen sections were taken through the entire nucleus accumbens. After three $10 \mathrm{~min}$ rinses in PBS, sections were incubated in either primary antibody to c-Fos (1:6000 in PBS with $0.3 \%$ Triton X-100; Santa Cruz Biotechnology, Santa Cruz, CA) or in primary antibody to calbindin-D (28 kDa) (1:6000 in PBS with $0.3 \%$ Triton $\mathrm{X}-100$; Chemicon International, Temecula, CA) at $4^{\circ} \mathrm{C}$ for $48 \mathrm{hr}$. Both the c-Fos and calbindin-D sections were then incubated for $45 \mathrm{~min}$ at room temperature in biotinylated anti-rabbit $\mathrm{IgG}$ secondary antibody (1:200 in PBS; Elite Vectastain ABC kit; Vector Laboratories, Burlingame, CA), followed by an incubation with an avidin-biotin horseradish peroxidase complex (1:50 in PBS; Elite Vectastain ABC kit) for $45 \mathrm{~min}$ at room temperature, with three 10 min rinses in PBS preceding each incubation. After two rinses in PBS and a $10 \mathrm{~min}$ rinse in $0.1 \mathrm{M}$ Tris buffer, $\mathrm{pH}$ 7.6, the c-Fos and calbindin-D sections were incubated for 5 and $10 \mathrm{~min}$, respectively, in $0.08 \%$ diaminobenzidine (DAB) (Aldrich, Milwaukee, WI) in Tris buffer containing $0.003 \%$ hydrogen peroxide and $0.015 \%$ nickel chloride. All sections were rinsed again in Tris buffer and deionized water and then mounted onto chrom-alum-coated slides. The slides were dried, dehydrated, cleared, and coverslipped using Permount (Fisher Scientific, Pittsburgh, PA)

Microscopic analysis. The neural tissue stained for calbindin-D, which delineates the shell and core of the nucleus accumbens (Jongen-Relo et al., 1994a; Johnson and Wood, 1999) was used to identify one section each at the rostral, middle, and caudal level of the dorsal nucleus accumbens. Sections from the nucleus accumbens at the rostral, middle, and caudal levels stained for calbindin are shown in Figure $1 A-C$. It has been reported that there are less distinct differences between the core and shell in calbindin-D immunoreactivity in the Syrian hamster compared with the rat, but staining for this peptide is still able to demarcate the subregions of the nucleus accumbens (Johnson and Wood, 1999). A box encompassing a sampling region of $0.1 \mathrm{~mm}^{2}(0.2 \times 0.5 \mathrm{~mm})$ was placed over the dorsal shell and core of the nucleus accumbens for each section. An image of each section was printed onto transparency film and the images were then superimposed onto the corresponding c-Fos sections for each animal, ensuring that the box was placed in the same position for all the animals. Figure $1, D$ and $E$, illustrates one caudal section from an animal that received 6 weeks of sexual experience and was tested for sexual behavior. The box was placed in the core of the caudal nucleus accumbens in Figure $1 D$ and in the shell of the caudal accumbens in Figure $1 E$. A box with the same dimensions was placed in the same tissue section in the medial cingulate cortex and over the medial and lateral dorsal caudate nucleus at each of the three levels sampled for the nucleus accumbens. Because we hypothesized that there could be rostral-caudal variations in the effects of mating on c-Fos, only one section per level was analyzed to increase the anatomical precision of our sampling. The number of c-Fos-immunoreactive cells in each selected area was counted with the aid of a video camera connected to a computerized image analysis system (BioQuant MegM; R \& M Biometrics, Nashville, TN).

\section{Experiment 1}

The first experiment investigated the effects of sexual experience and testing on c-Fos induction in the nucleus accumbens, dorsal caudate nucleus, and cingulate cortex. The goal of the experiment was twofold. The first goal was to determine whether there were differences in cellular activation in any of the brain regions because of previous sexual experience and/or behavioral testing. If c-Fos expression was altered, it was then determined whether the changes could be localized to specific subregions within the three brain areas analyzed.

Female Syrian hamsters received 6 weeks of sexual experience or remained sexually naive. During the 6 weeks of experience, the cumulative amount of time that the female assumed lordosis (immobility accompanied by a dorsoflexion of the back) was measured for each 10 min test session. No measures of male sexual behavior were recorded. During week 7, the same series of estradiol benzoate and progesterone injections were given. This time, half of the sexually experienced and naive females were tested for sexual behavior by placing an adult male in their home cage. The remaining females were left in their home cages. At 60-90 min after exposure to the male, the females were intracardially perfused, and their brains were processed for c-Fos expression. Those females not tested for sexual behavior were perfused $4 \mathrm{hr}$ after progesterone administration.

Data analysis. Because the cell counts did not differ between those females not tested for sexual behavior during week 7, regardless of past sexual experience (see Table 1 for an example), the experimental females were ultimately divided into three treatment groups for analysis. The first group contained the females that received 6 weeks of sexual experience and were tested for sexual behavior (experience/test, $n=6$ ). The second group consisted of those females that did not receive any previous experience, but were tested for sexual behavior (no experience/test, $n=$ 8 ). The final group contained all of the female hamsters that were not tested for sexual behavior, regardless of any previous sexual experience (no test, $n=13$ ). The two groups not receiving sexual behavior testing were combined to increase statistical power in the analyses. The number of c-Fos-stained cells from the dorsal nucleus accumbens, dorsal caudate nucleus, and cingulate cortex were compared among the three groups.

Cell counts were analyzed using multifactor ANOVAs. Simple maineffect ANOVAs and post hoc Newman-Keuls tests were performed 

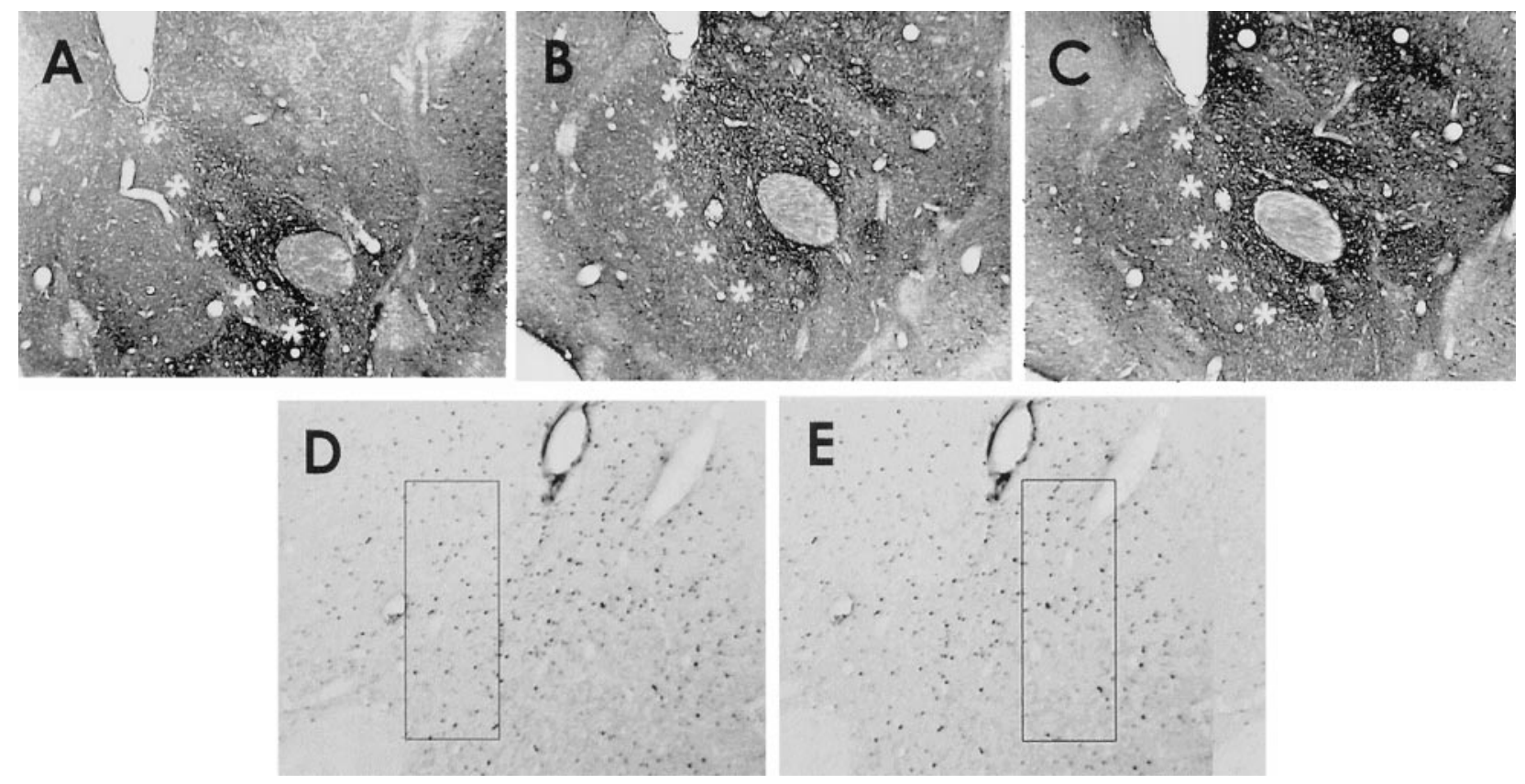

Figure 1. Nucleus accumbens tissue sections stained for calbindin-D and c-Fos. $A-C$ are sections from the rostral $(A)$, middle $(B)$, and caudal $(C)$ nucleus accumbens stained (midline is left) for calbindin, illustrating the division between the shell and core subregions (asterisks). There are $320 \mu \mathrm{m}$ between the rostral and middle sections and $240 \mu \mathrm{m}$ between the middle and caudal sections. The bottom images $(D, E)$ are examples of c-Fos staining from the caudal core $(D)$ and shell $(E)$ of the nucleus accumbens (midline is right) of a sexually experienced female killed after a sexual behavior test. The rectangle illustrates the sampling area $(0.2 \times 0.5 \mathrm{~mm})$.

Table 1. Comparison of mean \pm SEM number of c-Fos-immunoreactive cells in the nucleus accumbens shell and core between the no test treatment groups

\begin{tabular}{llr} 
& \multicolumn{2}{l}{ Treatment group } \\
\cline { 2 - 3 } Anatomical division & $\begin{array}{l}\text { Experience/ } \\
\text { no test }\end{array}$ & $\begin{array}{l}\text { No experience/ } \\
\text { no test }\end{array}$ \\
\hline $\begin{array}{l}\text { Nucleus accumbens shell } \\
\text { Rostral }\end{array}$ & $29.50 \pm 5.8$ & $36.14 \pm 5.6$ \\
$\quad$ Middle & $25.67 \pm 2.7$ & $24.57 \pm 3.8$ \\
$\quad$ Caudal & $15.50 \pm 3.3$ & $28.86 \pm 5.0$ \\
Nucleus accumbens core & $19.17 \pm 4.5$ & $23.71 \pm 5.4$ \\
$\quad$ Rostral & $19.67 \pm 2.8$ & $23.0 \pm 4.9$ \\
$\quad$ Middle & $18.33 \pm 2.9$ & $19.86 \pm 3.1$ \\
Caudal & & \\
\hline
\end{tabular}

The number of c-Fos-stained cells was compared between the two control no test treatment groups (experience/no test and no experience/no test). A three-way ANOVA (treatment times rostral-caudal level times shell-core) failed to indicate a significant three-way interaction and significant main effects of the treatment groups in either the shell or core of the accumbens, or through the rostral-caudal dimensions of the nucleus.

where appropriate. Behavioral data (lordosis durations) were analyzed using a two-tailed $t$ test.

\section{Experiment 2}

The second experiment compared the ability of a novel stimulus, amphetamine, to produce behavioral sensitization in sexually experienced and sexually naive female hamsters. c-Fos expression in the nucleus accumbens, dorsal caudate nucleus, and cingulate cortex was analyzed again to determine whether the pattern of cellular activity was similar to the results obtained in Experiment 1.

Female Syrian hamsters were given 6 weeks of sexual experience or remained sexually naive. At week 7, all females were transported to a novel environment (i.e., a 10 gallon glass aquarium in an unfamiliar room) $4 \mathrm{hr}$ after progesterone administration. The females were placed in the 10 gallon glass aquarium for $10 \mathrm{~min}$, after which time half of the sexually experienced and sexually naive females were administered D-amphetamine sulfate $(1 \mathrm{mg}$ per $1 \mathrm{~kg}$ body weight in $1.0 \mathrm{ml}$ of $0.9 \%$ $\mathrm{NaCl}$; a gift from Dr. David Nichols, Purdue University). The remaining females were injected with $0.9 \% \mathrm{NaCl}(1 \mathrm{mg}$ per $1 \mathrm{~kg}$ body weight). The females were then placed back into the 10 gallon aquarium for an additional $60 \mathrm{~min}$. The $70 \mathrm{~min}$ sessions were videotaped for analysis of the general locomotor activity of the females. Within 30 min after the general activity test, the females were intracardially perfused, and their brains were processed for c-Fos expression.

Videotape analysis. During week 7, the 70 min sessions testing locomotor activity were videotaped. The 10 gallon glass aquariums were divided into three equal areas on the video screen, and the locomotor activity of the females was recorded in terms of the number of area crosses.

Data analysis. Past sexual history did not influence the locomotor activity of the female hamsters injected with saline; therefore, the experimental females were divided into three treatment groups for analysis. The first group contained the females that received 6 weeks of sexual experience and were injected with amphetamine (experience/amphetamine, $n=8$ ). The second group consisted of those females that were administered amphetamine but did not receive any sexual experience (no experience/amphetamine, $n=8$ ). The final group contained all of the female hamsters that were injected with saline, regardless of any previous sexual experience (saline, $n=15$ ). The mean locomotor activity of the females was compared among the three treatment groups across the 70 min of testing (in 10 min periods) using two-factor ANOVAs. Simple main-effect ANOVAs and post hoc Newman-Keuls tests were performed where appropriate.

The number of cells stained for c-Fos did not differ between those females injected with saline, regardless of past sexual experience. Therefore, the number of c-Fos-stained cells from the dorsal nucleus accumbens, dorsal caudate nucleus, and cingulate cortex were compared among the same three treatment groups as in the first experiment. Cell counts were analyzed using multifactor ANOVAs. Simple main-effect ANOVAs and post hoc Newman-Keuls tests were performed where appropriate.

\section{RESULTS}

\section{Experiment 1}

\section{Sexual behavior measures}

The lordosis durations during the test for sexual behavior on week 7 were compared between the experience/test and the no 

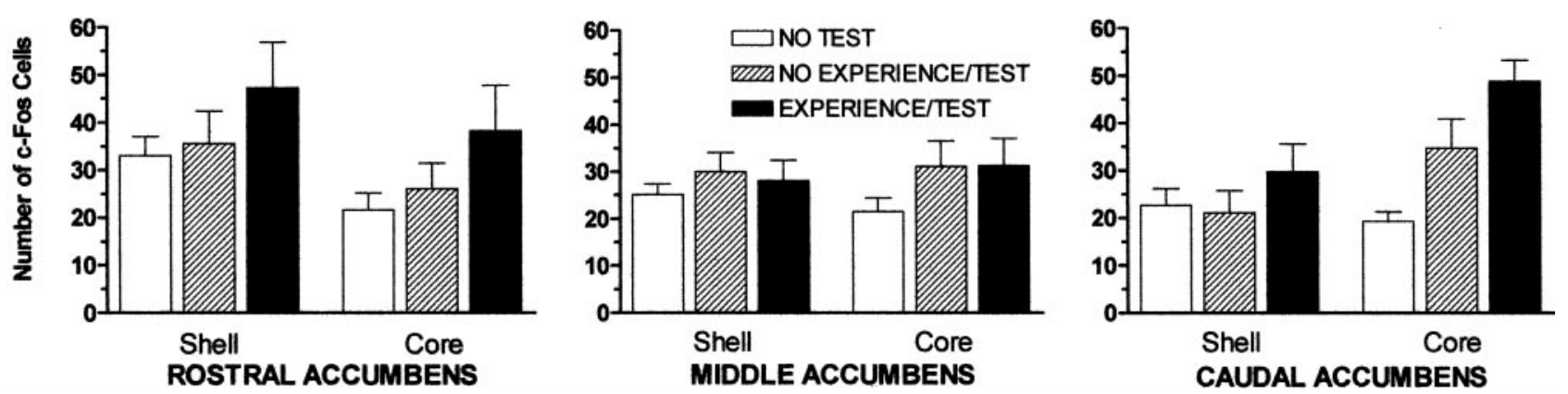

Figure 2. c-Fos expression in the shell and core of the nucleus accumbens at the rostral, middle, and caudal levels for each of the treatment groups. A three-way ANOVA (treatment times rostral-caudal level times shell-core) was used to examine the effects of sexual experience and behavior on the mean \pm SEM number of c-Fos cells. No significant main effects of treatment and no three-way interaction among treatment, accumbens level, and shell-core were found.

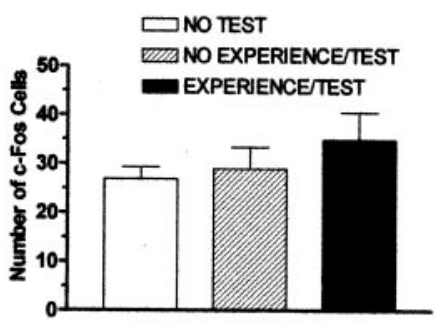

SHELL ACCUMBENS

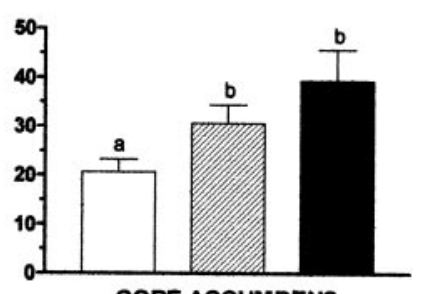

CORE ACCUMBENS

Figure 3. c-Fos expression in the shell and core of the nucleus accumbens, collapsed across the rostral-caudal level. The three-way ANOVA revealed a two-way interaction between treatment and mean \pm SEM number of c-Fos cells in the shell and core of the nucleus accumbens (treatment times shell-core; $F_{(2,24)}=4.243 ; p<0.026$ ). A one-way ANOVA probing this interaction found significant main effects of the treatment groups only in the nucleus accumbens core $\left(F_{(2,24)}=7.341 ; p<\right.$ $0.003)$ and not in the shell of the accumbens $\left(F_{(2,24)}=1.271 ; p>0.1\right)$. Different letters indicate significant differences between groups.

experience/test groups. The average lordosis duration during the $10 \mathrm{~min}$ test was $341 \pm 53 \mathrm{sec}$ for the experience/test group and $478 \pm 20 \mathrm{sec}$ for the no experience/test group. Females in the no experience/test group had assumed lordosis for a significantly longer duration than had the females in the experience/test group $\left(t_{6}=5.131 ; p=0.05\right)$. Furthermore, sexual experience did not affect lordosis duration. The analysis showed no significant differences between the average durations for week 1 (399 $\pm 44 \mathrm{sec})$ and week $7(341 \pm 53 \mathrm{sec})$ for females in the experience/test group.

\section{c-Fos expression in the nucleus accumbens}

A three-way ANOVA of treatment times rostral-caudal level times shell-core found no significant main effects of treatment and no three-way interaction among treatment, accumbens level, and shell-core (Fig. 2); however, two important two-way interactions (treatment times shell-core and treatment times rostralcaudal level) were detected.

Probing of the treatment times shell-core interaction revealed significant main effects of the treatment groups only in the core of the nucleus accumbens (Fig. 3). Pairwise multiple comparisons showed that those females tested for sexual behavior during week 7 (experience/test and no experience/test) had significantly more c-Fos-stained cells in the core of the nucleus accumbens than did those females that were not tested (no test) (Newman-Keuls, $p<$ 0.01). No effects of testing were observed in the shell of the nucleus accumbens. Furthermore, there were no explicit effects of

sexual experience on the number of cells expressing c-Fos in either the shell or core of the accumbens.

Probing of the treatment times rostral-caudal level interaction found significant main effects of the treatment groups in both the rostral and caudal levels but not in the middle level of the nucleus accumbens (Fig. 4). Newman-Keuls post hoc tests indicated that females that had received 6 weeks of sexual experience and were tested for sexual behavior (experience/test) had more c-Fospositive cells in the rostral nucleus accumbens than females that were tested but that did not receive any previous sexual experience (no experience/test; $p<0.05$ ) and those females that were not tested for sexual behavior (no test; $p<0.01$ ). The post hoc tests revealed similar results for the caudal nucleus accumbens. Females in the experience/test group had a higher number of cells expressing c-Fos in the caudal nucleus accumbens than females in the no experience/test group $(p<0.05)$ and no test group $(p<$ $0.01)$. Therefore, testing for sexual behavior during week 7 had increased the number of c-Fos-stained cells in the rostral and caudal nucleus accumbens only for those females that had received the 6 weeks of experience.

\section{c-Fos expression in the caudate nucleus and cingulate cortex}

Cell counts from the dorsal caudate nucleus were also analyzed using a three-way ANOVA. The analysis revealed only an interaction between treatment and c-Fos expression in the medial and lateral caudate nucleus $\left(F_{(2,24)}=3.514 ; p<0.046\right)$. However, separate analysis of the medial and lateral caudate nucleus by one-way ANOVA indicated no difference in the number of c-Fosstained cells between the experience/test, no experience/test, and no test groups (Table 2). In addition, no main effects of sexual experience or behavior on the number of cells expressing c-Fos, or any interactions, were found in the cingulate cortex (data not shown).

\section{Experiment 2}

Locomotor activity

A two-way ANOVA (treatment times testing period), comparing the mean activity of the females in the experience/amphetamine, no experience/amphetamine, and saline treatment groups across the $70 \mathrm{~min}$ of testing revealed an interaction between treatment group and testing period. To examine this interaction, the individual treatment groups were probed separately with one-way ANOVAs. The analyses indicated significant changes in the mean general activity during the 70 min of testing for the two groups of females that were injected with amphetamine (experience/amphetamine and no experience/amphetamine). However, the gen- 

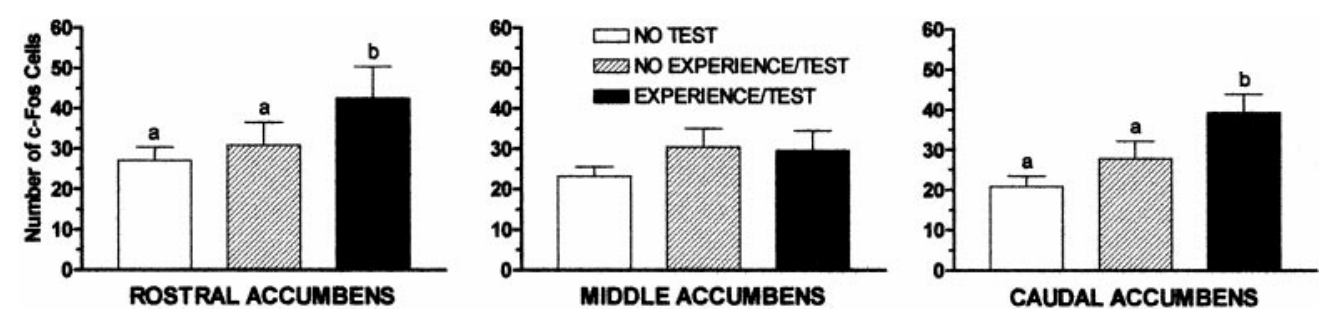

Figure 4. c-Fos expression through the rostral-caudal dimension of the nucleus accumbens, collapsed across the core and shell. Although a three-way ANOVA indicated that the two-way interaction between treatment groups and the mean \pm SEM number c-Fos cells through the rostral-caudal levels of the nucleus accumbens only approached significance $\left(F_{(4.48)}=2.365 ; p<0.066\right)$, we probed each level of the nucleus accumbens separately for an effect of treatment on c-Fos staining. A one-way ANOVA revealed significant main effects of the treatment groups in both the rostral level $\left(F_{(2,48)}=5.230\right.$; $p<0.009)$ and caudal level $\left(F_{(2,48)}=7.455 ; p<0.002\right)$ but not in the middle level $\left(F_{(2,48)}=1.744 ; p>0.1\right)$ of the nucleus accumbens. Different letters indicate significant differences between groups.

Table 2. Mean \pm SEM number of c-Fos-immunoreactive cells in the medial and lateral dorsal caudate nucleus

\begin{tabular}{llll} 
& \multicolumn{2}{l}{ Treatment group } \\
\cline { 2 - 4 } Anatomical division & $\begin{array}{l}\text { Experience/ } \\
\text { test }\end{array}$ & $\begin{array}{l}\text { No experience/ } \\
\text { test }\end{array}$ & No test \\
\hline Medial caudate nucleus & & & \\
$\quad$ Rostral & $35.67 \pm 7.0$ & $27.63 \pm 5.6$ & $20.77 \pm 2.4$ \\
$\quad$ Middle & $35.17 \pm 4.9$ & $34.75 \pm 6.9$ & $22.69 \pm 3.2$ \\
Caudal & $39.67 \pm 7.9$ & $29.88 \pm 7.4$ & $25.31 \pm 4.4$ \\
Lateral caudate nucleus & & & \\
$\quad$ Rostral & $24.17 \pm 5.9$ & $9.75 \pm 3.0$ & $16.15 \pm 2.9$ \\
$\quad$ Middle & $20.33 \pm 5.7$ & $19.25 \pm 4.2$ & $17.62 \pm 2.6$ \\
Caudal & $17.50 \pm 4.8$ & $17.00 \pm 7.8$ & $17.46 \pm 4.5$ \\
\hline
\end{tabular}

Analysis of the dorsal caudate nucleus found no significant treatment effects across either the rostral-caudal or medial-lateral dimensions of the nucleus.

eral activity of the females that received saline did not change significantly in the 70 min (Fig. 5). Newman-Keuls post hoc tests were then used to determine which 10 min testing periods differed. Pairwise multiple comparisons revealed that the general activity of the sexually experienced females given amphetamine significantly increased $10 \mathrm{~min}$ after injections $(p<0.05)$. Furthermore, compared with the 10 min before injections, females in the experience/amphetamine treatment group remained significantly more active $20 \mathrm{~min}(p<0.05)$ and $30 \mathrm{~min}(p<0.05)$ after injections. In contrast, the effects of amphetamine in sexually naive females were not evident until 20 min after injections. At this time point, these females were significantly more active compared with the 10 min before injections $(p<0.05)$. In addition, the activity of the sexually naive females given amphetamine remained significantly increased for $30 \mathrm{~min}(p<0.05)$ and $40 \min (p<0.01)$ after injections.

\section{c-Fos expression}

A three-way ANOVA (treatment times rostral-caudal level times shell-core) was used to examine the effects of sexual experience and amphetamine on c-Fos expression in the nucleus accumbens. No significant main effects of treatment and no three-way interaction among treatment, accumbens level, and shell-core were found. Furthermore, the analysis did not reveal any interactions between the treatment groups and c-Fos expression in the shell and core of the accumbens or between the treatment groups and c-Fos labeling in the rostral, middle, and caudal levels of the nucleus accumbens (data not shown).

Cell counts from the dorsal caudate nucleus were also analyzed using a three-way ANOVA. Initial analysis revealed no significant effect of previous sexual experience or amphetamine on the number of c-Fos-positive cells. In addition, no effect of previous sexual experience or amphetamine on the number of cells expressing c-Fos was found in the cingulate cortex using a two-way ANOVA (data not shown).

\section{DISCUSSION}

The purpose of this investigation was twofold. We first examined the effects of sexual experience on cellular activity in different subregions of the nucleus accumbens. The second issue, whether previous sexual experience could sensitize the mesolimbic dopamine pathway, was investigated by comparing the behavioral responses of sexually experienced and naive animals to an amphetamine injection. Our findings not only indicate that female sexual behavior can activate neurons in the nucleus accumbens but also that sexual experience can cross-sensitize neuronal responses to amphetamine.

\section{Sexual behavior effects on c-Fos expression in the shell and core of the nucleus accumbens}

Sexual behavior testing increased c-Fos expression in the core, but not the shell, of the nucleus accumbens, supporting previous research showing that a single sexual encounter can activate neurons in the nucleus accumbens in female rodents (Meisel et al., 1993; Joppa et al., 1995; Mermelstein and Becker, 1995; Pfaus et al., 1995; Kohlert et al., 1997; Kohlert and Meisel, 1999). Literature addressing the functional dichotomy of the nucleus accumbens is composed of numerous reports of differential changes in dopamine transmission within the shell and core of the nucleus accumbens in response to pharmacological and physiological stimuli. Administration of several drugs of abuse results in selective increases in extracellular dopamine levels in the shell of the nucleus accumbens (Pontieri et al., 1995; Nisell et al., 1997; Pierce and Kalivas, 1997a; Tanda et al., 1997; Tanda and Di Chiara, 1998; Barrot et al., 1999; Cadoni and Di Chiara, 1999). In a similar manner, highly palatable foods (Tanda and Di Chiara, 1998; Di Chiara et al., 1999a; Kelley, 1999), mild stress (e.g., foot shock) (Kalivas and Duffy, 1995; Tidey and Miczek, 1997; Bruijnzeel et al., 1999; Wu et al., 1999), and environmental novelty (Rebec et al., 1997; Rebec, 1998) also selectively increase dopamine transmission in the shell of the nucleus accumbens.

Our findings are consistent with the hypothesis that the shell and core are functionally distinct, although we found the core, and not the shell, to be responsive to sexual behavior. It is possible, however, that there were changes in c-Fos immunoreactivity in the shell of the nucleus accumbens, but these changes 

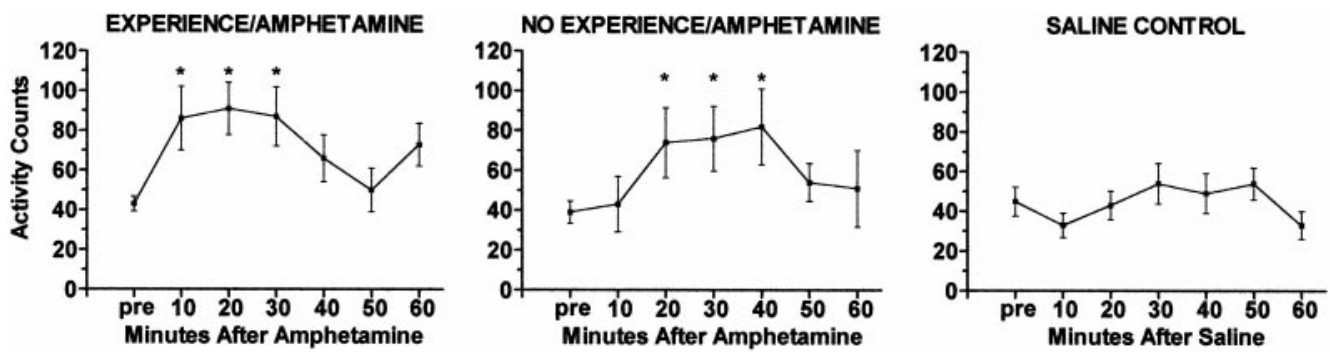

Figure 5. Effects of amphetamine on the general activity of sexually experienced and sexually naive female hamsters. A two-way ANOVA (treatment times testing period) revealed an interaction between treatment group and testing period $\left(F_{(12,150)}=2.288 ; p<0.011\right)$ for mean \pm SEM activity counts. A one-way ANOVA probing the individual treatment groups showed significant changes in the general activity for females in the experience/ amphetamine $\left(F_{(6,150)}=3.0468 ; p<0.008\right)$ and no experience/amphetamine $\left(F_{(6,150)}=3.893 ; p<0.001\right)$ treatment groups. The activity of the females injected with saline did not change $\left(F_{(6,150)}=1.619 ; p<0.1\right)$. Post hoc tests indicated that the sexually experienced females responded more rapidly to amphetamine, showing an increase in activity within the first 10 min after injection. Sexually naive females did not respond to the amphetamine until 20 min after injection. ${ }^{*} p<0.05$ versus the period before testing.

were not detected. The shell is complexly organized into different subregions, a medial, ventral, and lateral shell, with the ventral and dorsal areas of the medial shell possibly being two more distinct subregions (Groenewegen et al., 1999). These subregions of the shell, as well as the medial and lateral parts of the core, receive different combinations of inputs from cortical and subcortical areas (Groenewegen et al., 1999). Furthermore, located within these subregions are functionally distinct ensembles of neurons that are organized into distinct anatomical compartments (Groenewegen et al., 1999). Because the effects of sexual behavior on c-Fos expression in this study were examined in only the dorsomedial shell, it is possible that the number of c-Fospositive cells actually changed in a different shell subregion.

Despite the observations that many nucleus accumbens functions are localized to the shell region, it is reasonable to hypothesize that different neural circuits within the nucleus accumbens mediate the reinforcing properties of different behaviors. Carelli et al. (2000) have reported recently that nucleus accumbens neurons in rats exhibit similar neuronal activity during operant responding to two natural reinforcers (i.e., food and water) but different firing patterns during responding for a natural reinforcer versus cocaine. They have concluded that separate neural circuits in the nucleus accumbens process information about food and water reinforcement versus cocaine reward (Carelli et al., 2000).

\section{Sexual experience effects on c-Fos expression through the rostral-caudal axis of the nucleus accumbens}

The literature examining the subnuclear organization through the rostral-caudal nucleus accumbens is small; however, clear functional and anatomical differences have been observed. Our findings are consistent with studies that have reported differential regulation of neurochemical and motor responses across the rostral-caudal axis of the accumbens. Cholecystokinin (CCK) differentially modulates dopamine-induced effects in the rostral and caudal nucleus accumbens (Crawley et al., 1985a,b), potentiating dopamine-induced hyperlocomotion when infused into the caudal accumbens, a region innervated by CCK neurons colocalized with dopamine (Crawley et al., 1985a,b; Lanca et al., 1998). However, CCK is behaviorally inactive when injected into the rostral nucleus accumbens, a region that receives separate CCK and dopamine projections (Crawley et al., 1985a,b; Lanca et al., 1998). It has also been reported that direct infusion of amphetamine into the rostral shell, caudal shell, or core differentially affects behavioral activity and extracellular dopamine and serotonin levels (Heidbreder and Feldon, 1998). Regulation of opioid peptides, substance $\mathrm{P}$, dopamine $\mathrm{D} 1$ receptors (Voorn and Docter, 1992; Jongen-Relo et al., 1994b; Voorn et al., 1994), and acetylcholine release (Jongen-Relo et al., 1995) by dopamine and dopamine receptor agonists also differs between the rostral and caudal parts of the accumbens, with the rostral accumbens being more sensitive to dopamine depletion and administration. Although these functional differences between the rostral and caudal nucleus accumbens have been reported, why these functional differences exist is still not fully understood.

\section{Sexual experience effects on amphetamine-induced locomotor activity}

Results reported here and in an earlier study suggest that previous sexual experience sensitizes neuronal responses to sexual behavior testing, indicating sensitized increases in dopamine release (Kohlert and Meisel, 1999) and cellular activity in the nucleus accumbens (this study). One concern, however, is that experienced females in the previous study may have responded to both sexual behavior testing and environmental cues because sexual experience and testing were conducted in the same room. Environmental cues conditionally associated with motivated behaviors can acquire incentive properties and further increase dopamine levels in the nucleus accumbens (Reid et al., 1996, 1998; Watson and Little, 1999). A second concern is that because measures of male sexual behavior were not recorded, it is not known whether the two groups of females tested for sexual behavior received comparable amounts of vaginocervical stimulation. It has been reported that vaginocervical stimulation is necessary for dopamine release in the nucleus accumbens during mating (Kohlert et al., 1997). Perhaps the sexually experienced females received more vaginocervical stimulation (not measured in this study), thus increasing c-Fos induction. Therefore, to validate that female sexual behavior sensitizes the mesolimbic dopamine pathway, we investigated whether sexually experienced and naive females responded differently to an amphetamine injection, another stimulus known to mediate its effects via dopamine pathways. Furthermore, to ensure that the sensitized responses observed were because of repeated sexual behavior and not because of conditioned association of the environment to sexual behavior, the behavioral responses of the hamsters to amphetamine were tested in a novel environment.

Amphetamine increased general activity in all female hamsters. However, sexually experienced females responded sooner to amphetamine than did sexually naive females. These results validate the hypothesis that repeated sexual behavior can sensi- 
Table 3. Mean \pm SEM basal number of c-Fos-immunoreactive cells in the nucleus accumbens shell and core for control animals in experiments 1 and 2

Control group

\begin{tabular}{lrr}
\cline { 2 - 3 } Anatomical division & Experiment 1 & Experiment 2 \\
\hline $\begin{array}{l}\text { Nucleus accumbens shell } \\
\text { Rostral }\end{array}$ & $33.08 \pm 4.0$ & $65.67 \pm 4.6$ \\
Middle & $25.08 \pm 2.3$ & $77.67 \pm 4.8$ \\
$\quad$ Caudal & $22.69 \pm 3.6$ & $72.33 \pm 5.8$ \\
Nucleus accumbens core & & \\
$\quad$ Rostral & $21.62 \pm 3.5$ & $53.33 \pm 4.5$ \\
Middle & $21.46 \pm 2.9$ & $52.93 \pm 3.8$ \\
Caudal & $19.15 \pm 2.1$ & $61.0 \pm 4.2$
\end{tabular}

Although it is not statistically appropriate to compare the levels of c-Fos expression between the control animals in experiments 1 and 2, it is clear that there are two- to three-fold higher baseline cell counts in experiment 2 .

tize neurons in the mesolimbic dopamine pathway and suggest that changes in the pathway produce sensitized behavioral responses both to the natural motivated behavior and to a psychomotor stimulant (cross-sensitization).

These findings are consistent with the hypothesis that there are convergent neural mechanisms mediating responses to drugs and sexual behavior (Robinson and Berridge, 1993; Pierce and Kalivas, 1997b). Several recent studies have observed crosssensitization between repeated drug exposure and natural motivated behaviors. Social defeat stress reduces the acquisition time for cocaine self-administration in rats (Tidey and Miczek, 1997). An environment paired with repeated morphine injections can facilitate sexual behavior in male rats (Mitchell and Stewart, 1990a,b). Amphetamine pretreatment also facilitates sexual behavior in sexually naive male rats and is correlated with augmented dopamine release in the nucleus accumbens (Fiorino and Phillips, 1999).

c-Fos expression was analyzed in the nucleus accumbens after amphetamine treatment. It was hypothesized that amphetamine would increase c-Fos expression in the nucleus accumbens, and to a greater extent in sexually experienced females. However, no effects of amphetamine on the number of cells expressing c-Fos were found in any of the subregions of the nucleus accumbens. It is evident from Table 3 that the control animals in experiment 2 (saline females) had a higher number of c-Fos-positive cells compared with control animals in experiment 1 (no test females). Badiani et al. (1998) reported that novelty increased $c$-fos mRNA content in the nucleus accumbens, and that this effect of novelty on $c$-fos content was so strong in several brain regions that the administration of amphetamine in a novel environment did not produce an additional incremental response. Thus, it seems possible that in our study the stress of being transported to the novel environment of the testing room activated synthesis of the c-Fos protein, thereby masking changes in c-Fos expression induced by amphetamine and sexual experience.

\section{Potential significance}

These experiments join a growing list of studies (Mitchell and Stewart, 1990b; Fiorino and Phillips, 1999; Miczek et al., 1999) indicating that the experiences of an animal can sensitize the responsiveness of the mesolimbic dopamine pathway both to behaviors that are part of the natural repertoire of an animal and to certain drugs that humans are known to abuse (Wise and Bozarth, 1987). A key issue in research on drug abuse is individ- ual vulnerability to the effects of drugs (Newcomb, 1992; Robinson and Berridge, 1993), and collectively this research may offer insights into the development of addiction in people.

\section{REFERENCES}

Badiani A, Oates MM, Day HEW, Watson SJ, Akil H, Robinson TE (1998) Amphetamine-induced behavior, dopamine release, and c-fos mRNA expression: modulation by environmental novelty. J Neurosci 18:10579-10593.

Barrot M, Marinelli M, Abrous DN, Rouge-Pont F, Le Moal M, Piazza PV (1999) Functional heterogeneity in dopamine release and in the expression of Fos-like proteins within the rat striatal complex. Eur J Neurosci 11:1155-1166.

Bassareo V, Di Chiara G (1999) Differential responsiveness of dopamine transmission to food-stimuli in nucleus accumbens shell/core compartments. Neuroscience 89:637-641.

Birrell CE, Balfour DJK (1998) The influence of nicotine pretreatment on mesoaccumbens dopamine overflow and locomotor responses to D-amphetamine. Psychopharmacology 140:142-149.

Bradberry CW, Barrett-Larimore RL, Jatlow P, Rubino SR (2000) Impact of self-administered cocaine and cocaine cues on extracellular dopamine in mesolimbic and sensorimotor striatum in rhesus monkeys. J Neurosci 20:3874-3883.

Broening HW, Pu C, Vorhees CV (1997) Methamphetamine selectively damages dopaminergic innervation to the nucleus accumbens core while sparing the shell. Synapse 27:153-160.

Brog JS, Salyapongse A, Deutch AY, Zahm DS (1993) The patterns of afferent innervation of the core and shell in the "accumbens" part of the rat ventral striatum: immunohistochemical detection of retrogradely transported fluoro-gold. J Comp Neurol 338:255-278.

Brown P, Molliver ME (2000) Dual serotonin (5-HT) projections to the nucleus accumbens core and shell: relation of the 5-HT transporter to amphetamine-induced neurotoxicity. J Neurosci 20:1952-1963.

Bruijnzeel AW, Stam R, Compaan JC, Croiset G, Akkermans LMA, Olivier B, Wiegant VM (1999) Long-term sensitization of Fosresponsivity in the rat central nervous system after a single stressful experience. Brain Res 819:15-22.

Cadoni C, Di Chiara G (1999) Reciprocal changes in dopamine responsiveness in the nucleus accumbens shell and core and in the dorsal caudate-putamen in rats sensitized to morphine. Neuroscience 90:447-455.

Cadoni C, Solinas M, Di Chiara G (2000) Psychostimulant sensitization: differential changes in accumbal shell and core dopamine. Eur J Pharmacol 388:69-76.

Carelli RM, Ijames SG, Crumling AJ (2000) Evidence that separate neural circuits in the nucleus accumbens encode cocaine versus "natural" (water and food) reward. J Neurosci 20:4255-4266.

Crawley JN, Hommer DW, Skirboll LR (1985a) Topographical analysis of nucleus accumbens sites at which cholecystokinin potentiates dopamine-induced hyperlocomotion in the rat. Brain Res 335:337-341.

Crawley JN, Stivers JA, Blumstein LK, Paul SM (1985b) Cholecystokinin potentiates dopamine-mediated behaviors: evidence for modulation specific to a site of coexistence. J Neurosci 5:1972-1983.

Cunningham ST, Kelley AE (1992) Evidence for opiate-dopamine crosssensitization in nucleus accumbens: studies of conditioned reward. Brain Res Bull 29:675-680.

Di Chiara G, Loddo P, Tanda G (1999a) Reciprocal changes in prefrontal and limbic dopamine responsiveness to aversive and rewarding stimuli after chronic mild stress: implications for the psychobiology of depression. Biol Psychiatry 46:1624-1633.

Di Chiara G, Tanda G, Bassareo V, Pontieri F, Acquas E, Fenu S, Cadoni C, Carboni E (1999b) Drug addiction as a disorder of associative learning. Ann NY Acad Sci 877:461-485.

Fiorino DF, Phillips AG (1999) Facilitation of sexual behavior and enhanced dopamine efflux in the nucleus accumbens of male rats after D-amphetamine-induced behavioral sensitization. J Neurosci 1999:456-463.

Groenewegen HJ, Wright CI, Beiger AVJ, Voorn P (1999) Convergence and segregation of ventral striatal inputs and outputs. Ann NY Acad Sci 877:49-63.

Heidbreder C, Feldon J (1998) Amphetamine-induced neurochemical and locomotor responses are expressed differentially across the anteroposterior axis of the core and shell subterritories of the nucleus accumbens. Synapse 29:310-322.

Heimer L, Zahm DS, Churchill L, Kalivas PW, Wohltmann C (1991) Specificity in the projection patterns of accumbal core and shell in the rat. Neuroscience 41:89-125.

Heimer L, Alheid GF, de Olmos JS, Groenewegen HJ, Haber SN, Harlan RE, Zahm DS (1997) The accumbens: beyond the core-shell dichotomy. J Neuropsychiatry Clin Neurosci 9:354-381.

Ikemoto S, Panksepp J (1999) The role of nucleus accumbens dopamine in motivated behavior: a unifying interpretation with special reference to reward-seeking. Brain Res Brain Res Rev 31:6-41. 
Johnson LR, Wood RI (1999) The ventral striatum of the Syrian hamster. Ann NY Acad Sci 877:661-666.

Jongen-Relo AL, Voorn P, Groenewegen HJ (1994a) Immunohistochemical characterization of the shell and core territories of the nucleus accumbens in the rat. Eur J Neurosci 6:1255-1264.

Jongen-Relo AL, Docter GJ, Jonker AJ, Vreugdenhil E, Groenewegen HJ, Voorn P (1994b) Differential-effects of dopamine depletions on the binding and messenger-RNA levels of dopamine-receptors in the shell and core of the rat nucleus accumbens. Mol Brain Res 25:333-343.

Jongen-Relo AL, Docter GJ, Jonker AJ, Voorn P (1995) Differential localization of mRNAs encoding dopamine D1 or D2 receptors in cholinergic neurons in the core and shell of the rat nucleus accumbens. Mol Brain Res 28:169-174.

Joppa MA, Meisel RL, Garber MA (1995) c-Fos expression in female hamster brain following sexual and aggressive behaviors. Neuroscience 68:783-792.

Kalivas PW, Duffy P (1993) Time course of extracellular dopamine and behavioral sensitization to cocaine. I. Dopamine axon terminals. J Neurosci 13:266-275.

Kalivas PW, Duffy P (1995) Selective activation of dopamine transmission in the shell of the nucleus accumbens by stress. Brain Res 675:325-328

Kalivas PW, Striplin CD, Steketee JD, Klitenick MA, Duffy P (1992) Cellular mechanisms of behavioral sensitization to drugs of abuse. Ann NY Acad Sci 654:128-135.

Kelley AE (1999) Functional specificity of ventral striatal compartments in appetitive behaviors. Ann NY Acad Sci 877:71-90.

Kelley AE, Smith-Roe SL, Holahan MR (1997) Response-reinforcement learning is dependent on $N$-methyl-D-aspartate receptor activation in the nucleus accumbens core. Proc Natl Acad Sci USA 94:12174-12179.

Kohlert JG, Meisel RL (1999) Sexual experience sensitizes matingrelated nucleus accumbens dopamine responses of female Syrian hamsters. Behav Brain Res 99:45-52.

Kohlert JG, Rowe RK, Meisel RL (1997) Intromissive stimulation from the male increases extracellular dopamine release from fluoro-goldidentified neurons within the midbrain of female hamsters. Horm Behav 32:143-154.

Koob GF (1999) The role of the striatopallidal and extended amygdala systems in drug addiction. Ann NY Acad Sci 877:445-460.

Kuczenski R, Segal DS, Todd PK (1997) Behavioral sensitization and extracellular dopamine responses to amphetamine after various treatments. Psychopharmacology 134:221-229.

Lanca AJ, de Cabo C, Arifuzzaman AI, Vaccarino FJ (1998) Cholecystokinergic innervation of nucleus accumbens subregions. Peptides 19:859-868.

Lorrain DS, Arnold GM, Vezina P (1999) Mesoaccumbens dopamine and the self-administration of amphetamine. Ann NY Acad Sci 877:820-822.

Maldonado-Irizarry CS, Swanson CJ, Kelley AE (1995) Glutamate receptors in the nucleus accumbens shell control feeding behavior via the lateral hypothalamus. J Neurosci 15:6779-6788.

McKinzie DL, Rodd-Henricks ZA, Dagon CT, Murphy JM, McBride WJ (1999) Cocaine is self-administered into the shell region of the nucleus accumbens in Wistar rats. Ann NY Acad Sci 877:788-791.

Meisel RL, Camp DM, Robinson TE (1993) A microdialysis study of ventral striatal dopamine during sexual behavior in female Syrian hamsters. Behav Brain Res 55:151-157.

Mermelstein PG, Becker JB (1995) Increased extracellular dopamine in the nucleus accumbens and striatum of the female rat during paced copulatory behavior. Behav Neurosci 109:354-365.

Miczek KA, Mutschler NH, van Erp AMM, Blank AD, McInerney SC (1999) D-Amphetamine "cue" generalizes to social defeat stress: behavioral sensitization and attenuated accumbens dopamine. Psychopharmacology 147:190-199.

Mitchell JB, Gratton A (1994) Involvement of mesolimbic dopamine neurons in sexual behaviors: implications for the neurobiology of motivation. Annu Rev Neurosci 5:317-329.

Mitchell JB, Stewart J (1990a) Facilitation of sexual behaviors in the male rat associated with intra-VTA injections of opiates. Pharmacol Biochem Behav 35:643-650.

Mitchell JB, Stewart J (1990b) Facilitation of sexual behaviors in the male rat in the presence of stimuli previously paired with systemic injections of morphine. Pharmacol Biochem Behav 35:367-372.

Newcomb MD (1992) Understanding the multidimensional nature of drug use and abuse: the role of consumption, risk factors, and protective factors (Glantz M, Pickens R, eds), pp 255-297. Washington, DC: American Psychological Association.

Nisell M, Marcus M, Nomikos GG, Svensson TH (1997) Differential effects of acute and chronic nicotine on dopamine output in the core and shell of the rat nucleus accumbens. J Neural Transm 104:1-10.

Peoples LL, Uzwiak AJ, Gee F, Fabbricatore AT, Muccino KJ, Mohta BD, West MO (1999) Phasic accumbal firing may contribute to the regulation of drug taking during intravenous cocaine selfadministration sessions. Ann NY Acad Sci 877:781-787.

Pfaus JG, Damsma G, Wenkstern D, Fibiger HC (1995) Sexual activity increases dopamine transmission in the nucleus accumbens and striatum of female rats. Brain Res 693:21-30.

Pierce RC, Kalivas PW (1995) Amphetamine produces sensitized increases in locomotion and extracellular dopamine preferentially in the nucleus accumbens shell of rats administered repeated cocaine. J Pharmacol Exp Ther 275:1019-1029.

Pierce RC, Kalivas PW (1997a) Repeated cocaine modifies the mechanism by which amphetamine releases dopamine. J Neurosci 17:3254-3261.

Pierce RC, Kalivas PW (1997b) A circuitry model of the expression of behavioral sensitization to amphetamine-like psychostimulants. Brain Res Brain Res Rev 25:192-216.

Pierre PJ, Vezina P (1998) $\mathrm{D}_{1}$ dopamine receptor blockade prevents the facilitation of amphetamine self-administration induced by prior exposure to the drug. Psychopharmacology 138:159-166.

Pontieri FE, Tanda G, Di Chiara G (1995) Intravenous cocaine, morphine, and amphetamine preferentially increase extracellular dopamine in the "shell" as compared with the "core" of the rat nucleus accumbens. Proc Natl Acad Sci USA 92:12304-12308.

Rebec GV (1998) Real-time assessments of dopamine function during behavior: single-unit recording, iontophoresis, and fast-scan cyclic voltammetry in awake, unrestrained rats. Alcohol Clin Exp Res 22:32-40.

Rebec GV, Christensen JRC, Guerra C, Bardo MT (1997) Regional and temporal differences in real-time dopamine efflux in the nucleus accumbens during free-choice novelty. Brain Res 776:61-67.

Reid MS, Ho LB, Berger SP (1996) Effects of environmental conditioning on the development of nicotine sensitization: behavioral and neurochemical analysis. Psychopharmacology 126:301-310.

Reid MS, Ho LB, Berger SP (1998) Behavioral and neurochemical components of nicotine sensitization following 15-day pretreatment: studies on contextual conditioning. Behav Pharmacol 9:137-148.

Robinson TE, Berridge KC (1993) The neural basis of drug craving: an incentive-sensitization theory of addiction. Brain Res Brain Res Rev 18:247-291

Robinson TE, Jurson PA, Bennett JA, Bentgen KM (1988) Persistent sensitization of dopamine neurotransmission in ventral striatum (nucleus accumbens) produced by prior experience with $(+)$-amphetamine: a microdialysis study in freely moving rats. Brain Res 462:211-222.

Salamone JD (1994) The involvement of nucleus accumbens dopamine in appetitive and aversive motivation. Behav Brain Res 61:117-133.

Salamone JD (1996) The behavioral neurochemistry of motivation: methodological and conceptual issues in studies of the dynamic activity of nucleus accumbens dopamine. J Neurosci Methods 64:137-149.

Stratford TR, Kelley AE (1997) GABA in the nucleus accumbens shell participates in the central regulation of feeding behavior. J Neurosci 17:4434-4440.

Tanda G, Di Chiara G (1998) A dopamine- $\mu 1$ opioid link in the rat ventral tegmentum shared by palatable food (Fonzies) and nonpsychostimulant drugs of abuse. Eur J Neurosci 10:1179-1187.

Tanda G, Pontieri FE, Di Chiara G (1997) Cannabinoid and heroin activation of mesolimbic dopamine transmission by a common $\mu_{1}$ opioid receptor mechanism. Science 276:2048-2050.

Taylor JR, Horger BA (1999) Enhanced responding for conditioned reward produced by intra-accumbens amphetamine is potentiated after cocaine sensitization. Psychopharmacology 142:31-40.

Tidey JW, Miczek KA (1997) Acquisition of cocaine self-administration after social stress: role of accumbens dopamine. Psychopharmacology 130:203-212.

Voorn P, Docter GJ (1992) A rostrocaudal gradient in the synthesis of enkephalin in nucleus-accumbens. NeuroReport 3:161-164.

Voorn P, Docter GJ, Jogen-Relo AL, Jonker AJ (1994) Rostrocaudal subregional differences in the response of enkephalin, dynorphin and substance-P synthesis in rat nucleus-accumbens to dopamine depletion. Eur J Neurosci 6:486-496.

Watson WP, Little HJ (1999) Prolonged effects of chronic ethanol treatment on responses to repeated nicotine administration: interactions with environmental cues. Neuropharmacology 38:587-595.

Wise RA, Bozarth MA (1987) A psychomotor stimulant theory of addiction. Psychol Rev 94:469-492.

Wu YL, Yoshida M, Emoto H, Tanaka M (1999) Psychological stress selectively increases extracellular dopamine in the "shell," but not in the "core" of the rat nucleus accumbens: a novel dual-needle probe simultaneous microdialysis study. Neurosci Lett 275:69-72.

Zahm DS (1999) Functional-anatomical implications of the nucleus accumbens core and shell subterritories. Ann NY Acad Sci 877:113-128.

Zahm DS, Brog JS (1992) On the significance of subterritories in the "accumbens" part of the rat ventral striatum. Neuroscience 50:751-767. 